\title{
Vulnerabilidade costeira frente a mudanças climáticas e políticas públicas na cidade do Rio de Janeiro: estamos prontos?
}

\section{Coastal Vulnerability in the face of climate change and public policies in the city of Rio de Janeiro: Are we prepared?}

Thaís Morais Corrêa Borges de Aguiar - Graduada em Administração, pela Universidade do Estado do Rio de Janeiro (UERJ), com MBA em Gestão de Projetos, pela Anhanguera, e MBA em Gestão do Conhecimento, pela Unyleya. E-mail: thaismcbaguiar@hotmail.com

Manuela Ervatti - Graduada em Administração, pela Universidade do Estado do Rio de Janeiro (UERJ). E-mail: maervatti@gmail.com

\begin{abstract}
Resumo
No cenário mundial concernente às mudanças climáticas, em que o município do Rio de Janeiro é visto como uma região de alto potencial de risco e vulnerabilidade costeira, a cidade passa a estar exposta com mais recorrência a eventos extremos e a implantação de medidas para a mitigação de tais efeitos é necessária. O presente artigo tem como objetivos, a partir do conceito de vulnerabilidade, identificar as áreas mais afetadas da cidade e investigar as políticas públicas existentes quanto aos possíveis riscos ambientais, em decorrência das mudanças climáticas globais, com ênfase nas zonas mais vulneráveis da região costeira. A pesquisa é desenvolvida por meio de uma abordagem qualitativa e procedimentos de pesquisa documental. $\mathrm{Na}$ conclusão, são apresentadas sugestões para o desenvolvimento de novas pesquisas voltadas para vulnerabilidade costeira local, ampliando o leque de intervenção para além da perspectiva mundial, com referência à problemática das mudanças climáticas.
\end{abstract}

\section{Palavra-chave}

Gestão Ambiental. Mudanças Climáticas. Vulnerabilidade Costeira. Rio de Janeiro. Políticas Públicas.

\begin{abstract}
Regarding the climate change in the global scene, in which Rio de Janeiro is seen as a region of high potential of risk and coastal vulnerability, the city turns out to be more exposed to extreme events. Seen in these terms, it's increasingly necessary the implementation of measures in order to mitigate these effects. Based on the concept of coastal vulnerability, this article has the objective to identify the most affected areas of the city and to investigate the policies concerning the ambiental risks, due to the global climate change phenomenon, that has been occurring with particular emphasis on the most vulnerable zones of the city's coastal region. The research has been developed through a qualitative approach by using documentary research procedures. In conclusion, suggestions are presented in order to develop new researches focused on the local intervention concerning coastal vulnerability, beyond the already existent global policies.
\end{abstract}

\section{Keywords}

Environmental Management. Climate Change. Coastal Vulnerability. Rio de Janeiro. Public Policies. 


\section{INTRODUÇÃO}

As mudanças climáticas são efeitos do aquecimento global e são visíveis em escala mundial (ANDRADE; LACERDA, 2009; BINDOFF et al., 2007 apud GERMANI et al., 2015). Além disso, efeitos como a acentuação do El Niño, os desequilíbrios na chuva e as enchentes são também fenômenos que podem ser observados no mundo todo (GERMANI et al., 2015). Dentre os impactos das mudanças climáticas previstos para o Brasil, estão o aumento de temperatura, as modificações nos padrões de chuvas, as alterações na distribuição de extremos climáticos tais como: secas, inundações, penetração de frentes frias, tempestades severas, vendavais e outros. Outrossim, o previsto aumento do nível médio do mar trará consequências para os ecossistemas e as populações que residem nas zonas costeiras e ribeirinhas que sofrem a influência das marés (NOBRE, 2001).

Nesse cenário, as regiões costeiras são vistas como vulneráveis ${ }^{1}$ a eventos extremos cada vez mais frequentes e intensos (GERMANI et al., 2015). Ademais, a intensa antropização dessa zona só agrava mais o efeito desses eventos (IPCC, 2001 apud GERMANI et al., 2015). Consequentemente, a população que vive nessas áreas está sujeita a acontecimentos como: tsunamis, marés meteorológicas e ondas extremas (KLEIN et al., 2009). Além disso, conforme a análise das empresas seguradoras, a zona costeira é considerada a de maior risco de ocupação mesmo sem levar em conta as mudanças climáticas (KRON, 2008 apud KLEIN et al., 2009).

Segundo Nicolodi e Pettermann (2010), o litoral da Região Metropolitana do Rio de Janeiro tem índice de vulnerabilidade muito alto se comparado a todo o litoral brasileiro, sendo um local exposto a frentes frias, tempestades vindas do sul e uma das áreas de maior risco a eventos extremos no país. O Rio de Janeiro é a maior aglomeração urbana da zona costeira brasileira e possui um numeroso contingente de população de baixa renda morando em áreas vulneráveis a esses eventos extremos no contexto do Atlântico Sul (UN-HABITAT, 2001 apud EGLER; GUSMÃO, 2014). Além disso, a cidade, desde o início da colonização portuguesa, se mostrou imprópria para habitação, tendo sido ocupada por meio da destruição de ecossistemas, que consequentemente levou a reações perigosas do meio ambiente (AMADOR, 2013).

Souza (2009) explica que as pressões socioeconômicas da zona costeira brasileira têm gerado uma intensa urbanização, sem que se observe o desgaste

A UNESCO (2009), por meio da Comissão Oceanográfica Intergovernamental (COI), define vulnerabilidade costeira como o estado das comunidades costeiras (incluindo estrutura social, ativos físicos, economia e suporte ambiental) que as torna mais ou menos suscetíveis a eventos extremos. 
dos recursos naturais. Sendo uma área de grande concentração populacional, a cidade do Rio de Janeiro está suscetível a problemas de erosão costeira, agravados por obras de engenharia que, por muitas vezes, não consideraram o equilíbrio morfológico da costa, gerando o desequilíbrio do balanço sedimentar (NEVES; MUEHE, 2008).

Sendo assim, é imprescindível que políticas públicas ambientais tenham foco na zona costeira por conta da vulnerabilidade que esta zona está sujeita frente às mudanças climáticas cada vez mais recorrentes (NICOLODI; PET'TERMANN, 2010). Dito isso, considerando a falta de conhecimento e base cartográfica sobre a zona costeira do Brasil (KLEIN et al., 2009), não apenas a questão ambiental deve ser observada nas discussões sobre desastres naturais, mas também as questões humanas e sociais, visto que os eventos extremos afetam a sociedade (VEYRET, 2007). Portanto, os estudos voltados para esses eventos na zona costeira do Rio de Janeiro devem ser concluídos em políticas públicas necessárias para a mitigação dos efeitos causados por tais eventos, visando proteger a população que vive em áreas de risco.

O desenvolvimento do artigo se dará a partir dos seguintes objetivos:

- Identificar, a partir do conceito de vulnerabilidade costeira, quais são os locais de maior vulnerabilidade na zona costeira do município do Rio de Janeiro; e

- Pesquisar políticas públicas e planos de adaptação existentes voltados para áreas costeiras de risco, e avaliar se são adequadas e suficientes.

\section{METODOLOGIA}

A metodologia utilizada no presente artigo tem abordagem qualitativa, natureza de pesquisa básica e procedimentos de pesquisa documental, que se assemelha a uma pesquisa bibliográfica, mas se baseia em documentos que ainda não receberam análise ou que podem ser reutilizados de acordo com cada pesquisa (GIL, 2002).

A pesquisa documental consiste no exame de diversos materiais que não foram utilizados para nenhum trabalho de análise, ou que podem ser reexaminados, buscando-se outras interpretações ou informações complementares, chamados de documentos (GUBA; LINCOLN, 1981).

O contexto escolhido para estudo foi a região costeira do município do Rio de Janeiro. Buscamos, por meio de dados documentais, uma melhor compreensão das áreas mais afetadas e das políticas públicas do Rio de Janeiro voltadas à vulnerabilidade costeira. 
Neste artigo, a partir do conceito de vulnerabilidade da Comissão Oceanográfica Intergovernamental (COI) - UNESCO (2009), do Intergovernmental Panel on Climate Change (IPCC, 2014) e autores como Dolan e Walker (2003 apud PIRES; CRAVEIRO; ANTUNES, 2012), verificamos quais as áreas de maior vulnerabilidade da região costeira do Rio de Janeiro e a gestão ambiental pública efetiva existente para essas áreas. Especificamente, pesquisamos os locais mais vulneráveis a eventos extremos na cidade e realizamos uma análise das políticas públicas ativas para tais áreas.

Com base nas políticas públicas pesquisadas sobre o assunto em tela, coletamos dados para os juízos de valor formulados na conclusão da pesquisa. São os dados coletados que auxiliam o pesquisador "a acompanhar o desenvolvimento do objeto ou fenômeno focalizado, a obter informações precisas sobre ele, e a tirar conclusões sobre determinadas características suas" (ELLIOT, 2012, p. 13).

\section{VULNERABILIDADE COSTEIRA}

A zona costeira possui inúmeros recursos naturais e diversidade ambiental, bem como enorme potencial para o desenvolvimento de diversas atividades econômicas (CARTER, 1990 apud GERMANI et al., 2015). Porém, é uma região em que os efeitos das mudanças climáticas têm a tendência de serem agravados por conta da forte antropização dos espaços urbanos, deixando as populações e instalações dessa zona em uma situação vulnerável (IPCC, 2001 apud GERMANI et al., 2015).

Nesse sentido, a vulnerabilidade da zona costeira pode ser entendida como a predisposição a um determinado risco, englobando diversos elementos, como a sensibilidade e a falta de capacidade de adaptação a um possível dano causado por esse risco (IPCC, 2014). Risco esse que pode ser dividido em risco natural, relacionado a processos naturais ou induzidos por meio do homem; tecnológico, relacionado aos processos produtivos e industriais; e social, resultante das desigualdades sociais que contribuem para a degradação das condições de vida de uma determinada população (NICOLODI; PETTTERMANN, 2010).

Portanto, as vulnerabilidades, com relação aos riscos ambientais, devem levar em consideração tanto fatores de ordem social, como de ordem econômica e geofísica (O'RIORDAN, 2000 apud PIRES; CRAVEIRO; ANTUNES, 2012).

É importante entender a vulnerabilidade como as circunstâncias históricas e condições sociais que colocam uma população em situação de risco devido a processos de erosão costeira (DOLAN; WALKER, 2003 apud PIRES; 
CRAVEIRO; ANTUNES, 2012). Grupos mais vulneráveis são aqueles que pela sua posição social e geográfica sofrem ou podem sofrer mais com os impactos negativos (CLARK et al., 1998 apud PIRES; CRAVEIRO; ANTUNES, 2012).

Logo, os estudos e avaliações da vulnerabilidade costeira são importantes porque além de serem instrumentos importantes para a prevenção de impactos socioeconômicos nas cidades litorâneas, também são imprescindíveis para o planejamento urbano e regional dessas áreas (RIBEIRO et al., 2013).

\section{LOCAIS DE MAIOR VULNERABILIDADE NO RIO DE JANEIRO}

O Rio de Janeiro éo estado do Brasil com a maior concentração populacional nos municípios da zona costeira. Sendo assim, a maioria da população está distribuída na extensa planície da costa, região muito atrativa para atividades turísticas nacionais e internacionais e portuárias. Além disso, no município do Rio de Janeiro estão presentes importantes complexos industriais petroquímicos, refinarias, centros de construções navais e bases militares (KLEIN et al., 2009).

Com relação à cidade do Rio de Janeiro é importante destacar que a orla apresenta diversas áreas vulneráveis a vários aspectos ambientais: praias, áreas sujeitas a alagamentos, brejos, lagoas, baías e outros. Ademais, encostas e planícies da zona costeira também são consideradas vulneráveis de acordo com a mudança de temperatura, ocupação da população e precipitação (KLEIN et al., 2009).

Pode-se dividir os locais de maior vulnerabilidade costeira da cidade de acordo com os eventos específicos de cada região. Procuramos ilustrar o trabalho utilizando como fonte os mapas do Instituto Nacional de Pesquisas Espaciais (INPE), além de Flood Maps (s.d.) e Klein et al. (2009).

\subsection{ZONAS COSTEIRAS DE BAIXA ELEVAÇÃO}

As Zonas Costeiras de Baixa Elevação (LECZ), que representam mais que 18\% do território da Região Metropolitana do Rio de Janeiro, são áreas que devem ser levadas em consideração, especialmente devido à grande ocupação populacional no litoral carioca. Além disso, as LECZ são áreas próximas à costa que têm altitude de até dez metros acima do mar e seus assentamentos urbanos configuram locais de risco devido ao grande potencial de alagamento (MANDARINO; ARUEIRA, 2012).

Na Figura 1, podem-se observar, como áreas vulneráveis na cidade do Rio de Janeiro, as seguintes regiões: Caju, Maré, Ilha do Fundão e Ilha do Governador. 
Figura 1 - Alagamento em LECZ no Rio de Janeiro

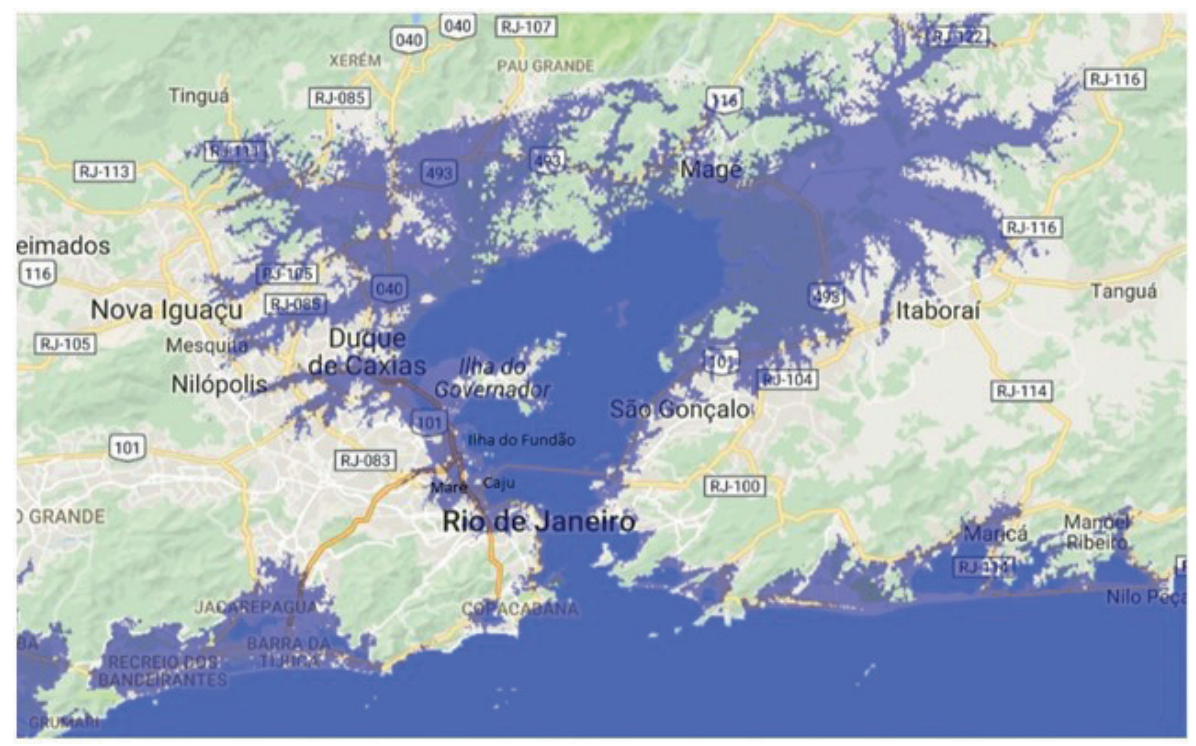

Fonte: Adaptado de Flood Maps (s.d.).

\subsection{PRAIAS OCEÂNICAS}

\subsubsection{Ondas}

Já as praias oceânicas, as mais vulneráveis quanto às ondas, são alteradas ajustando sua posição em planta e em perfil (KLEIN et al., 2009). Essa modificação pode ser observada na Figura 2.

Figura 2 - Exemplo de resposta da praia de Ipanema-Leblon a um clima de ondas de Sudeste

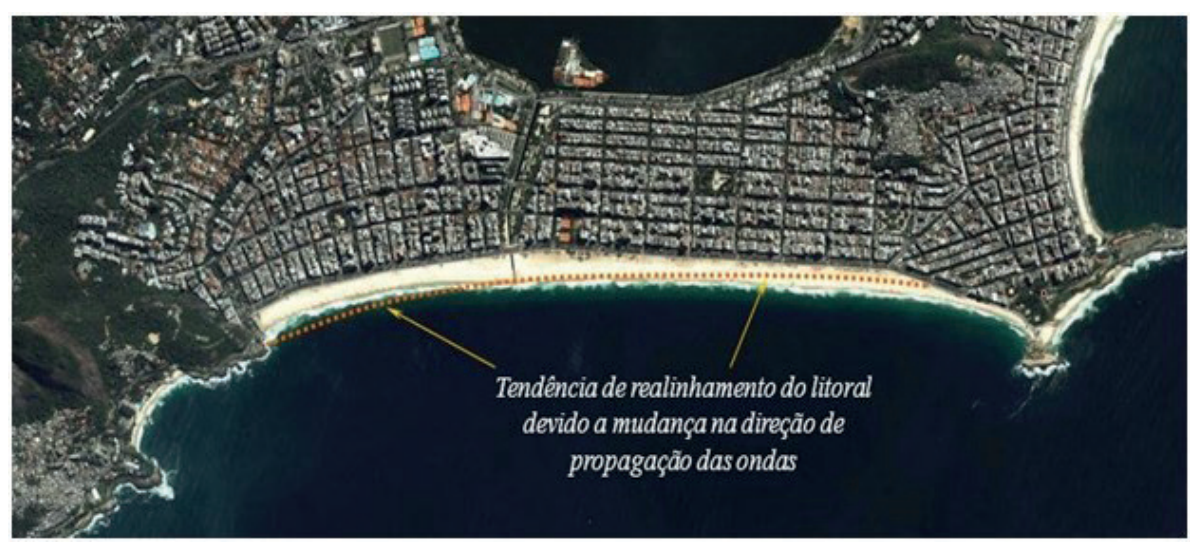

Fonte: Klein et al. (2009).

Novos Cadernos NAEA • v. 23 n. 2 • p. 161-178 • maio-ago 2020 
Por conta da ocupação indevida na faixa dinâmica das praias, muitos dos moradores estão suscetíveis a ondas de tempestades, visto que se tem pouco estoque de areia, causando alagamentos e erosão (KLEIN et al., 2009). Tais eventos tornam-se cada vez mais recorrentes com as mudanças climáticas globais, que tendem a elevar o nível do mar, aumentar o período de seca e tornar mais fortes as tempestades e os ventos vindos com elas. Nesses casos, é necessário o engordamento ${ }^{2}$ da praia (INPE, 2011).

Além disso, as ressacas têm acontecido com recorrência na cidade, expondo a costa oceânica a inundações nas avenidas e nos andares subterrâneos (RIO DE JANEIRO, 2018).

\subsubsection{Mudanças de ventos}

Praias como Ipanema, Arpoador e Leblon podem ter um desequilíbrio nos transportes de sedimentos causados pelas mudanças de ventos e climas de ondas, levando a um déficit de sedimentos no Arpoador e um acúmulo no Leblon. Eventos desse tipo tendem a se tornar recorrentes com as mudanças climáticas (INPE, 2011). Os ventos na zona costeira carioca podem gerar aumento do nível do mar, além de colocarem em risco as torres de transmissão e antenas de comunicação (RIO DE JANEIRO, 2018).

\subsection{LAGOAS}

Com relação às lagoas da cidade do Rio de Janeiro, significativamente alteradas pelo homem, a qualidade das suas águas está comprometida (KLEIN et al., 2009) e a tendência é que piore cada vez mais, tornando-se um problema de saúde pública. Além disso, há registros de grandes ressacas na cidade do Rio de Janeiro que também geraram inundações nas áreas do entorno às lagoas costeiras (RIO DE JANEIRO, 2018). Dentre as lagoas, as mais afetadas são: a lagoa Rodrigo de Freitas, que sofre alagamentos nos períodos de chuva intensa e corre riscos de inundações, e as lagoas da Baixada de Jacarepaguá, que são as mais vulneráveis à elevação do nível do mar (INPE, 2011). O sistema lagunar de Jacarepaguá tem seu espelho d'água se expandindo e atingindo áreas urbanas próximas, como os bairros Rio das Pedras, Vargens e Itanhangá (MANDARINO; ARUEIRA 2012).

2 Segundo o INPE (2011), o engordamento se dá por meio do aumento do estoque de areia nas praias. 


\subsection{BAÍAS}

\subsubsection{Ventos fortes e chuvas intensas}

Quanto às baías, a de Guanabara é um local onde houve modificações significativas por conta da urbanização e da criação de aterros. Além disso, essa baía está sujeita a ventos fortes acompanhados por chuvas intensas. Por isso, é necessária uma constante monitoração do regime de ventos, com vistas à observação de tendências decenais (KLEIN et al., 2009). para o controle do vento na região.

\subsubsection{Elevação do nível do mar}

A baía de Sepetiba, a Baixada Fluminense e o sistema lagunar de Jacarepaguá são os locais mais vulneráveis à elevação do nível do mar na cidade do Rio, conforme podemos observar na Figura 3. Destes, o caso mais grave é o da baía de Jacarepaguá, onde se localizam os bairros Itanhangá, Rio das Pedras e as Vargens, que são suscetíveis a constantes enchentes advindas do sistema lagunar de Jacarepaguá, as quais tendem a se tornar mais recorrentes com as mudanças climáticas (INPE, 2011).

Figura 3 - Áreas vulneráveis à elevação do nível do mar e consequente redefinição da linha de costa

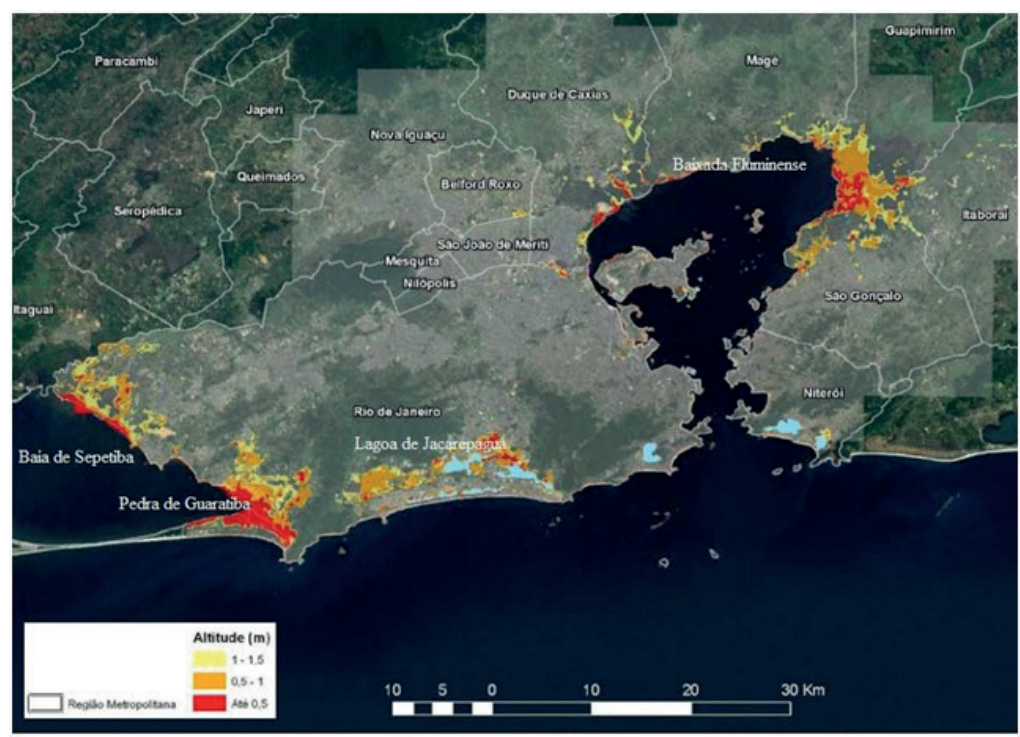

Fonte: Adaptado de INPE (2011). 


\subsubsection{Ondas}

Na baía de Sepetiba, em Pedra de Guaratiba, a construção de muros de contenção permitiu a recuperação de uma orla lamosa, porém não foram levadas em conta as ações de ondas, podendo se observar uma destruição dos muros. Implicações como esta podem ser resultado da falta de observação de aspectos essenciais na engenharia costeira, como estimativa da presença de ondas locais, cartografia e batimetria detalhadas e medição maregráfica. Em Sepetiba, a criação de muros de contenção tornou a situação da região mais crítica, com a destruição dos mesmos e a fuga do material de aterro (KLEIN et al., 2009).

Na baía de Guanabara há a necessidade de monitoramento da variação do nível do mar, que causa danos no quebra-mar da marina da Glória por conta das ondas de sudeste em épocas de ressaca. A orla voltada para leste da Ilha do Governador é suscetível a inundações provocadas por ondas geradas pelo vento nordeste (KLEIN et al., 2009).

\subsection{BREJOS E ÁREAS ALAGADIÇAS}

Em relação aos brejos e áreas alagadiças, os de situação mais crítica estão localizados na região da orla da baía de Sepetiba, Vargem Grande e Recreio dos Bandeirantes. Outra área da cidade vulnerável a inundações é a praça da Bandeira (KLEIN et al., 2009), que tem como causa de eventos extremos o aterramento de manguezais, várzeas e pântanos (SOUZA; OTTONI, 2015). É importante ressaltar que as inundações são processos naturais e essenciais para o sistema fluvial. Porém, a retirada da vegetação ripária, as construções em locais próximos aos rios e a impermeabilização do solo na área drenada são agravantes da situação (INPE, 2011).

\section{MEDIDAS DE ADAPTAÇÃO EXISTENTES}

Dentre os planos concernentes às mudanças climáticas, em âmbito nacional, podemos citar o Plano Nacional sobre Mudança no Clima (PNMC), criado em 2008 por meio do Decreto no 6.263, de 21 de novembro de 2007. O PNMC visa incentivar o desenvolvimento de medidas para reduzir as emissões de gases do efeito estufa e criar condições para que o país consiga lidar com os efeitos das mudanças climáticas, além de outras melhorias ambientais e socioeconômicas (BRASIL, 2007). 
Referente ao estudo da vulnerabilidade costeira, o plano cita as pesquisas do Instituto Nacional de Pesquisas Espaciais (INPE) relacionadas a esse tema, incluindo medidas de adaptação e mapeamento das zonas mais vulneráveis. Também define como um dos objetivos do programa de mudança do clima identificar quais as maiores vulnerabilidades na costa brasileira. Especificamente no tópico de estudos sobre impactos, vulnerabilidades e estratégias de adaptação, o PNMC apresenta um macrodiagnóstico da zona costeira, abrangendo características físico-naturais e socioeconômicas da costa brasileira com objetivo de "orientar ações de conservação, regulamentação e fiscalização dos patrimônios naturais e culturais" (BRASIL, 2007, p. 101). Consta a carta de risco à inundação da costa, que orienta as esferas regional, local e federal a tomarem decisões para a redução dos riscos a eventos extremos com medidas de prevenção e ações imediatas.

Ainda segundo o PNMC, foi desenvolvido pela Universidade Federal do Rio de Janeiro (UFRJ), por meio da Área de Engenharia Costeira \& Oceanográfica, do Programa de Engenharia Oceânica da COPPE e do Departamento de Recursos Hídricos e Meio Ambiente da Escola Politécnica, um estudo sobre vulnerabilidades no litoral do estado do Rio de Janeiro devido às mudanças climáticas. A pesquisa teve como objetivo principal debater as potenciais vulnerabilidades nas diversas áreas da zona costeira do estado do Rio de Janeiro por meio de análises e dados, resultando em previsões para 2050 e 2100 e sugerindo medidas para mitigação e adaptação às mesmas. O plano cita o cenário tendencioso de elevação do nível médio do mar, mudanças de direção de ondas e aumento de extremos climáticos (BRASIL, 2007).

Além do PNMC, no âmbito nacional também podemos citar o Plano Nacional de Adaptação à Mudança do Clima (PNA), criado em maio de 2016, por meio da Portaria no 150 , de 10 de maio de 2016. O PNA tem como objetivo promover a redução da vulnerabilidade nacional à mudança climática e realizar uma gestão do risco associada a esse fenômeno por meio da identificação da exposição do país a impactos atuais e futuros com base em estudos como a projeção do clima, identificação e análise da vulnerabilidade a esses impactos e, por fim, definição de ações e diretrizes para uma melhor adaptação. A elaboração do PNA levou em consideração 11 setores: Agricultura, Recursos Hídricos, Cidades, Gestão de Riscos e Desastres, Segurança Alimentar e Nutricional, Biodiversidade, Indústria e Mineração, Povos e Populações Vulneráveis, Infraestrutura, Zonas Costeiras e Saúde (BRASIL, 2016).

As estratégias do PNA para a adaptação nas zonas costeiras foram desenvolvidas após identificação da exposição da zona costeira brasileira à 
mudança climática, levando em conta os principais impactos e vulnerabilidades e indicando diretrizes, ações e metas necessárias para melhor estratégia de adaptação. O plano também deixa claro que as lacunas existentes no conhecimento sobre a zona costeira do Brasil contribuem muito para aumentar a vulnerabilidade do país e recomenda o estudo das regiões metropolitanas costeiras contemplando os aspectos socioeconômicos, ambientais e de infraestrutura, levando em consideração as mudanças climáticas (BRASIL, 2016).

Para trazer maior transparência e acompanhamento sistemático da estratégia nacional de adaptação, o governo federal desenvolveu, em 2017, o relatório de monitoramento e avaliação do primeiro ano de implementação do PNA informando a evolução das metas e diretrizes nacionais para adaptação, relatório que será desenvolvido anualmente. De acordo com o mesmo, nesse primeiro ano, o PNA contribuiu para a implementação do PNMC e para o fortalecimento de outras políticas públicas e marcos internacionais (BRASIL, 2017).

Das metas e diretrizes previstas no PNA, segundo o primeiro relatório de monitoramento e avaliação do período 2016-2017, foram realizadas iniciativas para 100\% e 67\% delas, respectivamente, tendo um total de 24 metas e 136 diretrizes. Quanto às medidas voltadas para a região costeira, o PNA iniciou as três metas previstas, e das 12 diretrizes realizou apenas 2 delas. Dentre as medidas realizadas, estão o Programa Nacional para Conservação da linha de Costa (PROCOSTA), a coalizão de instituições para o apoio de Núcleos de Referência para o Gerenciamento Costeiro e um critério regional para definição desses núcleos, a criação de grupo temático para elaboração de metodologia e levantamento de custos de compatibilização da altimetria continental com a batimetria, o inventário dos dados existentes dos macro diagnósticos da Zona Costeira e a cooperação para elaborar os requisitos do novo macro diagnóstico com uma plataforma com banco de dados e a aprovação da participação do Brasil na elaboração do próximo relatório do IPCC na parte referente a Oceanos e Criosfera. Segundo o relatório, os próximos desafios seriam adquirir recursos financeiros e humanos para a implementação das diretrizes e metas do setor e a criação de um plano para a implantação dos Núcleos de Referência para o Gerenciamento Costeiro (BRASIL, 2017).

No âmbito municipal, o Rio de Janeiro criou uma série de medidas para a mitigação dos efeitos das mudanças climáticas nos últimos anos. A cidade foi uma das primeiras do país a definir uma Política Municipal de Mudanças Climáticas e Desenvolvimento Sustentável, Lei nº 5.248, de 27 de janeiro de 2011 (RIO DE JANEIRO, 2011), por meio da qual definiu metas para redução da emissão dos gases do efeito estufa gradativamente desde 2012, com relação às emissões de 
2005. A próxima meta a ser alcançada é a de redução de $20 \%$ da emissão desses gases até o ano de 2020.

Com relação às áreas vulneráveis, essa política coloca como princípio "identificar vulnerabilidades e promover ações efetivas de adaptação aos impactos negativos da mudança do clima, protegendo principalmente as populações e ecossistemas mais vulneráveis" (RIO DE JANEIRO, 2011, p. 2). Além disso, coloca como diretriz:

promover pesquisas, produção e a divulgação de conhecimento sobre as mudanças climáticas e sobre as vulnerabilidades dela decorrentes, bem como para o estabelecimento de medidas de mitigação e adaptação das emissões de gases de efeito estufa no Município (RIO DE JANEIRO, 2011, p. 2).

Porém, especificamente em relação à vulnerabilidade costeira não há nada mencionado na lei.

Ademais, considerado um dos principais instrumentos na condução e consolidação dessa Política Municipal de Mudanças Climáticas e Desenvolvimento Sustentável, o Rio de Janeiro também possui o Fórum Carioca de Mudanças Climáticas e Desenvolvimento Sustentável, composto pelo Poder Público, iniciativa privada e sociedade civil com o objetivo de

conscientizar e mobilizar a sociedade e o governo do município do Rio de Janeiro para discutirem os problemas decorrentes das mudanças do clima e promover o desenvolvimento sustentável, contribuindo para o crescimento econômico, a preservação ambiental e o desenvolvimento social (RIO DE JANEIRO, 2009, p. 1).

Dentre as atribuições presentes no decreto está a de reunir propostas para a mitigação da emissão dos gases do efeito estufa. Porém, não há medidas sobre a vulnerabilidade costeira definidas no decreto do Fórum.

Também com foco na redução dos gases do efeito estufa, em 2010, se iniciou a elaboração do sistema de monitoramento pela prefeitura do Rio de Janeiro, a fim de acompanhar e medir as emissões e suas variações com cada medida de mitigação implementada desde 2005, e compará-las com as metas do cenário referencial e com o desempenho de outros países que adotaram o sistema (RIO DE JANEIRO, 2010).

Finalmente, com foco nas zonas mais vulneráveis quanto aos efeitos das mudanças climáticas, em 2011, a Secretaria Municipal de Meio Ambiente elaborou em parceria com o INPE o Mapa de Vulnerabilidade da Região Metropolitana do Rio de Janeiro (RMRJ), que apresenta os impactos das mudanças climáticas 
na RMRJ, os locais de maior vulnerabilidade socioeconômica e natural dessa região e, por fim, soluções de adaptação. O texto cita a maior frequência de tempestades como um dos principais motivos para o mapeamento das áreas vulneráveis, além da elevação do nível do mar, ondas de tempestade e ventos intensos, que causam inundações nos locais de maior vulnerabilidade. O mapa considera a distribuição populacional desigual como um fator agravante e as zonas costeiras de baixa elevação como as zonas mais vulneráveis a eventos extremos (INPE, 2011).

Na primeira parte do documento são ressaltadas as projeções das mudanças climáticas futuras, levando em conta a precipitação e as temperaturas máxima e mínima, e também se ressaltam os impactos na orla costeira e as consequências da elevação do nível médio do mar, das mudanças no clima de ondas e do aumento de extremos climáticos, além de ações de engenharia para prevenção e remediação e redefinição da linha de costa. Na segunda parte, constam impactos na biodiversidade, savanização da vegetação e vulnerabilidade dos manguezais e das lagoas e, na terceira parte, infraestrutura de drenagem urbana, saneamento ambiental, gestão dos resíduos sólidos e saúde pública (INPE, 2011).

Por fim, em 2013, foi publicado o terceiro Inventário das Emissões de Gases do Efeito Estufa do Rio de Janeiro e a atualização do plano municipal para redução das emissões. Quanto a estes, o texto frisa medidas municipais como a duplicação da malha cicloviária na cidade, a expansão do programa de reflorestamento, a instalação do centro de tratamento de resíduos, a queima de biogás, entre outros. Além disso, explica que por conta do desenvolvimento econômico e do crescimento demográfico, dentre outros fatores, as emissões quase dobraram de 2005 a 2012, apesar de, desde 2000 a cidade ter iniciado a elaboração dos inventários de emissões de gases estufa. Resumindo, o inventário apresenta uma visão geral das medidas públicas quanto às emissões e seus resultados (RIO DE JANEIRO, 2013).

Após o estudo das áreas costeiras mais vulneráveis do município do Rio de Janeiro e identificação das medidas existentes para a adaptação e redução dos efeitos das mudanças climáticas nessas zonas, verificamos que existem poucas políticas, no âmbito municipal, voltadas diretamente para zonas costeiras vulneráveis, tendo as mesmas como principal foco simplesmente a diminuição das emissões dos gases de efeito estufa.

É de fato importante que as medidas públicas tenham como proposta a redução da emissão dos gases de efeito estufa (EGLER, 2010). Com relação a isso, as medidas estudadas anteriormente, o PNMC, o PNA, a Política Municipal de Mudanças Climáticas e Desenvolvimento Sustentável, o Fórum Carioca de 
Mudanças Climáticas e Desenvolvimento Sustentável e o Inventário das Emissões de Gases do Efeito Estufa do Rio de Janeiro, apresentam expressa preocupação e foco nesse sentido.

Porém, também é necessária a criação de medidas mitigatórias no que diz respeito aos efeitos da elevação do nível do mar e de eventos extremos em áreas vulneráveis (EGLER, 2010). É imprescindível o desenvolvimento de uma gestão estratégica para a adaptação a eventos extremos e políticas públicas ambientais com foco na zona costeira brasileira no que tange às mudanças climáticas cada vez mais recorrentes na região (NICOLODI; PET'TERNANN, 2010). Quanto a isso, dentre as políticas estudadas, observamos esse foco somente no PNMC, no PNA e no Mapa de Vulnerabilidade da Região Metropolitana do Rio de Janeiro.

Além disso, dentre as medidas públicas estudadas no âmbito municipal, é evidente a falta de atualização e monitoramento da situação das áreas vulneráveis da cidade do Rio de Janeiro e o alto nível de incerteza a respeito dos possíveis eventos de risco.

A região costeira do município do Rio de Janeiro, local exposto a frentes frias e tempestades vindas dos sul, tem índice de vulnerabilidade muito alto se comparado a todo o litoral brasileiro, sendo uma das áreas de maior risco relacionado a eventos extremos no país (NICOLODI; PETTERMANN, 2010). Por ser a maior aglomeração urbana da costa brasileira e de extrema importância econômica e logística para o país (EGLER; GUSMÃO, 2014) a predisposição a riscos ambientais nessa área deve ser levada a sério, visando melhor preparo para a mitigação adequada desses riscos e adaptação aos possíveis danos.

\section{CONCLUSÃO}

Com base nos objetivos propostos, podemos destacar como principais conclusões positivas do presente trabalho, a existência, em âmbito nacional, de medidas atualizadas voltadas para as zonas costeiras e, além disso, medidas em longo prazo e em nível mundial já estão sendo tomadas e apenas precisam ter continuidade.

Em relação aos aspectos negativos, constatamos a necessidade de maior envolvimento do governo, população e iniciativa privada em políticas municipais voltadas especificamente para a vulnerabilidade costeira da cidade do Rio de Janeiro. Além disso, faltam recursos humanos e financeiros para apoiar a implantação de ações voltadas para as zonas costeiras, e existem grandes lacunas no mapeamento e estudo das áreas de maior vulnerabilidade e risco. Ademais, as políticas públicas municipais estudadas possuem um foco majoritário em redução 
de gases do efeito estufa e créditos de carbono por pressão internacional e dão pouca ênfase em políticas voltadas para áreas costeiras de risco.

Quanto a novos estudos, há uma demanda para medidas imediatas voltadas para a mitigação dos efeitos de eventos extremos, como inundações e ventos intensos, e soluções aplicáveis de curto prazo para as ondas de tempestades e mudanças na direção e elevação do nível do mar, bem como de soluções para o realinhamento das faixas de areia. Além disso, é importante que as pesquisas sejam focadas nas áreas costeiras locais com a observação das necessidades específicas para cada área vulnerável, que mudam de acordo com a região.

Nesse sentido, é necessária uma maior aproximação dos pesquisadores e dos órgãos públicos às populações vulneráveis, para um maior conhecimento das reais necessidades de cada local. Por fim, concluímos que são indispensáveis mais pesquisas voltadas diretamente para a vulnerabilidade costeira no Rio de Janeiro, visto que é um assunto urgente e pouco explorado, tendo em vista a necessidade da criação de novas políticas públicas a respeito do assunto.

\section{REFERÊNCIAS}

AMADOR, E. Baía de Guanabara. Ocupação histórica e avaliação ambiental. Rio de Janeiro: Editora Interciência, 2013.

ANDRADE, E. S. M.; LACERDA, G. B. M. Mudanças climáticas e análise de risco da indústria de petróleo no litoral brasileiro. Madrid: Fundación Mapfre, 2009. Disponível em: http://www.mapfre.com/ documentacion/publico/118n/ catalogo_imagenes/grupo.cmd?path=1053958. Acesso em: 03 ago. 2018.

BINDOFF, N. L. et al. Observations: oceanic climate change and sea level. In: SOLOMON, S. et al. (org.). Climate change 2007: the physical science basis. Contribution of working group I to the Fourth Assessment Report of the Intergovernmental Panel on Climate Change. Cambridge: Cambridge University Press, 2007. p. 385-432. Disponível em: http://www.ipcc.ch/pdf/. Acesso em: 03 ago. 2018.

BRASIL. Decreto n ${ }^{\circ}$ 6.263, de 21 de novembro de 2007. Institui o Comitê Interministerial sobre Mudança do Clima - CIM, orienta a elaboração do Plano Nacional sobre Mudança do Clima, e dá outras providências. Diário Oficial da União, Brasília, DF, 22 de nov. de 2007. Seção 1, p. 5.

BRASIL. Ministério do Meio Ambiente. Portaria no 150, de 10 de maio de 2016. Institui o Plano Nacional de Adaptação à Mudança do Clima e dá outras providências. Diário Oficial da União, Brasília, DF, 11 de maio de 2016. Seção 1, p. 131. 
BRASIL. Ministério do Meio Ambiente. Plano Nacional de Adaptação à Mudança do Clima: $1^{\circ}$ relatório de monitoramento e avaliação 2016 - 2017. Brasília, DF, MMA, 2017.

EGLER, C. O Rio de Janeiro e as mudanças climáticas globais: uma visão geoeconômica. Claudio A. Egler - Geoeconomia, Rio de Janeiro, 2010. Desenvolvimento Regional. Disponível em: http://www.egler.com.br/pdf/ visao_geoeconomica_egler2_1.pdf. Acesso em: 30 jul. 2018.

EGLER, C.; GUSMÃO, P. P. Gestão costeira e adaptação às mudanças climáticas: o caso da Região Metropolitana do Rio de Janeiro, Brasil. Revista da Gestão Costeira Integrada, Lisboa, v. 14, n. 1, p. 65-81, mar. 2014. Disponível em: http:/ / www.scielo.mec.pt/pdf/rgci/v14n1/v14n1a06.pdf. Acesso em: 10 out. 2018.

ELLIOT, L. (org.). Instrumentos de avaliação e pesquisa: caminhos para construção e validação. Rio de Janeiro: Wak Editora, 2012.

FLOOD MAPS. South America. Flood Maps, [S. l.], [s.d.]. Disponível em: http:// flood.firetree.net/?ll=-24.5271,-62.2265\&z=14\&m=7. Acesso em: 10 out. 2018.

GERMANI, Y. et al. Vulnerabilidade costeira e perda de ambientes devido à elevação do nível do mar no litoral sul do Rio Grande do Sul. Revista da Gestão Costeira Integrada, Lisboa, v. 15, n. 1, p. 121-131, mar. 2015. Disponível em: http:/ /www. scielo.mec.pt/pdf/rgci/v15n1/v15n1a10.pdf. Acesso em: 26 out. 2018.

GIL, A. C. Como elaborar projetos de pesquisa. 4. ed. São Paulo: Atlas, 2002. GUBA, E.; LINCOLN, Y. Effective Evaluation. São Francisco: Jossey-Bass, 1981.

INPE. Região Metropolitana do Rio de Janeiro e as vulnerabilidades às mudanças climáticas. Rio de Janeiro: INPE, 2011. Disponível em: http:// www.rio.rj.gov.br/dlstatic/10112/2122393/DLFE-232588.pdf/mapa_ vulnerabilidade_inpe_smac.pdf. Acesso em: 02 nov. 2018.

IPCC. Climate change 2014: impacts, adaptation, and Vulnerability - part B: regional aspects. New York: Cambridge University Press, 2014. Disponível em: https://www.ipcc.ch/site/assets/uploads/2018/02/WGIIAR5-PartB_FINAL. pdf. Acesso em: 14 nov. 2018.

KLEIN, A. H. F. et al. Relatório de vulnerabilidade da Zona Costeira Brasileira às mudanças climáticas. Rio de Janeiro: COPPE/UFRJ, 2009. Disponível em: http://eadterrazul.org.br/pdf/Documentos/Oficina $\% 20$ de $\% 20$ Planejamento $\% 20-\% 20$ SCSLJ / 2\%C2\%AA \%20dia \%20-29.08.14\%20 Saneamento $\% 20$ B $\%$ C3\%A1 sico/Relat $\%$ C3\%B3rio_Vulnerabilidade-ZonaCosteira_Brasileira_v4.pdf. Acesso em: 26 out. 2018. 
MANDARINO, F.; ARUEIRA, L. Vulnerabilidade à elevação do nível médio do mar na Região Metropolitana do Rio de Janeiro. Coleção Estudos Cariocas, Rio de Janeiro, n. 20120702, p. 2-15, 2012. Disponível em: http:// portalgeo.rio.rj.gov.br/estudoscariocas/download\%5C3207_Vulnerabilidade aelevacaodonivelmediodomarna_RMRJ.pdf. Acesso em: 13 set. 2020.

NEVES, C. F.; MUEHE, D. Vulnerabilidades, impactos e adaptação a mudanças do clima: a zona costeira. Parcerias Estratégicas, Brasília, DF, n. 27, p. $217-$ 295, dez. 2008. Disponível em: http://seer.cgee.org.br/index.php/parcerias_ estrategicas/article/viewFile/325/319. Acesso em: 10 de set. 2020.

NICOLODI, J. L.; PETTTERMANN, R. M. Mudanças climáticas e a vulnerabilidade da Zona Costeira do Brasil: Aspectos ambientais, sociais e tecnológicos. Revista da Gestão Costeira Integrada, Lisboa, v. 10, n. 2, p. 151 177, 2010. Disponível em: http://www.redalyc.org/pdf/3883/388340129001. pdf. Acesso em: 30 jul. 2018.

NOBRE, C. A. Mudanças climáticas globais: possíveis impactos nos ecossistemas do país. Parcerias Estratégicas, Brasília, DF, v. 6, n. 12, p. 239-258, set. 2001. Disponível em: http://seer.cgee.org.br/index.php/parcerias_estrategicas/ article/view/186. Acesso em: 15 ago. 2018.

PIRES, I.; CRAVEIRO, J.; ANTUNES, O. Artificialização do solo e vulnerabilidade humana em duas zonas sujeitas a processos de erosão costeira: casos de estudo da Costa da Caparica e Espinho (Portugal). Revista da Gestão Costeira Integrada, Lisboa, v. 12, n. 3, p. 277-290, set. 2012. Disponível em: http://www.scielo.mec. pt/pdf/rgci/v12n3/v12n3a02.pdf. Acesso em: 14 nov. 2018.

RIBEIRO, J. S. et al. Evolução da vulnerabilidade à erosão costeira na Praia de Massaguaçú (SP), Brasil. Revista de Gestão Costeira Integrada, Lisboa, v. 13, n. 3, p. 253-265, set. 2013. Disponível em: http://www.scielo.mec.pt/pdf/rgci/ v13n3/v13n3a01.pdf. Acesso em: 19 nov. 2018.

RIO DE JANEIRO (Estado). Plano de Adaptação Climática do Estado do Rio de Janeiro: relatório final. Rio de Janeiro: Secretaria de Estado do Ambiente, 2018. 116 p. Disponível em: http://centroclima.coppe.ufrj.br/ images/documentos/Produto_11_PAERJ-Relat\%C3\%B3rio_Final.pdf. Acesso em: 22 out. 2018.

RIO DE JANEIRO (Município). Decreto no 31.415 de 30 de novembro de 2009. Institui o Fórum Carioca de Mudanças Climáticas e Desenvolvimento Sustentável. Diário Oficial do Município do Rio de Janeiro, Rio de Janeiro, RJ, 1 de dez. de 2009. p. 3. 
RIO DE JANEIRO (Município). Secretaria de Meio Ambiente da Cidade do Rio de Janeiro. Sistema de Monitoramento de Gases do Efeito Estufa. Prefeitura da Cidade do Rio de Janeiro, Rio de Janeiro, 26 jan. 2010. Disponível em: http://www.rio.rj.gov.br/web/smac/exibeconteudo?id=2812823. Acesso em: 29 nov. 2018.

RIO DE JANEIRO (Município). Lei no 5.248, de 27 de janeiro de 2011. Institui a Política Municipal sobre Mudança do Clima e Desenvolvimento Sustentável, dispõe sobre o estabelecimento de metas de redução de emissões antrópicas de gases de efeito estufa para o Município do Rio de Janeiro e dá outras providências. Diário Oficial do Município do Rio de Janeiro, Rio de Janeiro, RJ, 28 de jan. de 2011. p. 3.

RIO DE JANEIRO (Município). Secretaria de Meio Ambiente da Cidade do Rio de Janeiro. Inventário das emissões de gases de efeito estufa da cidade do Rio de Janeiro e atualização do Plano Municipal para Redução das Emissões. Rio de Janeiro, RJ: SMAC, 2013. Disponível em: http://www. rio.rj.gov.br/dlstatic/10112/1712030/4114528/CRJ_InventarioGEE2012_ resumo_tecnicoPORTUGUESFINAL.pdf. Acesso em: 13 nov. 2018.

SOUZA, C. R. G. A erosão costeira e os desafios da gestão costeira no Brasil. Revista da Gestão Costeira Integrada, Lisboa, v. 9, n. 1, p. 17-37, jan. 2009. Disponível em: https://www.redalyc.org/pdf/3883/388340125003.pdf. Acesso em: 11 de set. 2020.

SOUZA, T. M. K.; OTTONI, A. B. Análise Crítica das Causas e Soluções Sustentáveis para o Controle das Enchentes Urbanas: O Caso Prático da Bacia Hidrográfica da Praça da Bandeira. Revista Nacional de Gerenciamento de Cidades, São Paulo, v. 03, n. 17, p. 60-76, 2015. Disponível em: http://www. amigosdanatureza.org.br/publicacoes/index.php/gerenciamento_de_cidades/ article/view/1009/1032. Acesso em: 13 set. 2020.

UNESCO. Hazard awareness and risk mitigation in integrated coastal area management. Paris: UNESCO, 2009. 141 p. Disponível em: https://unesdoc. unesco.org/ark:/48223/pf0000183253. Acesso em: 31 out. 2018.

VEYRET, Y. (org.). Os riscos: o homem como agressor e vítima do meio ambiente. São Paulo: Contexto, 2007. 